\title{
AUMENTO DEL CONTENIDO DE ÁCIDOS HÚMICOS EN UN CARBÓN DE BAJO RANGO A TRAVÉS DE LA OXIDACIÓN CON AIRE Y CON PERÓXIDO DE HIDROGENO O ÁCIDO NÍTRICO
}

\author{
Ruben Anillo-Correa, Fredy Colpas-Castillo* y Edgardo Meza-Fuentes \\ Facultad de Ciencias Exactas y Naturales, Universidad de Cartagena, Colombia
}

Recebido em 30/4/12; aceito em 18/9/12; publicado na web em 23/1/13

\begin{abstract}
INCREASE OF THE CONTENT OF HUMIC ACIDS IN A LOW RANK COAL BY OXIDATION WITH AIR AND WITH HYDROGEN PEROXIDE OR NITRIC ACID. Low-rank coals are an important source of humic acids, which are important in retention processes of water and nutrients in plants. In this study coal samples of Montelibano, Colombia, were oxidized with air at different temperatures and subsequently with $\mathrm{H}_{2} \mathrm{O}_{2}$ and $\mathrm{HNO}_{3}$. The materials were characterized by FTIR, proximate and elemental analysis, and quantification of humic acids. The oxidation process led to an increased content of oxygenated groups and humic acids in the carbonaceous structure. The solid oxidized with air at $200{ }^{\circ} \mathrm{C}$ for $12 \mathrm{~h}$ and re-oxidized with $\mathrm{HNO}_{3}$ for $12 \mathrm{~h}$ showed the highest percentage of humic acids $(85.3 \%)$.
\end{abstract}

Keywords: coal; humic acids; oxidation.

\section{INTRODUCCIÓN}

La materia orgánica natural o humus contiene una fracción soluble en soluciones alcalinas llamadas sustancias húmicas, las cuales se forman por la descomposición del material vegetal y animal que se deposita en el suelo. Durante este proceso el material orgánico es inicialmente degradado y depolimerizado para posteriormente por acción microbiológica producir nuevos componentes con un alto grado de polimerización de coloración oscura y con un carácter no biodegradable con estructuras irregulares de alto peso molecular. ${ }^{1}$ Las sustancias húmicas se clasifican dependiendo del proceso de separación utilizado; los mayores componentes del humus son los ácidos fulvicos (AF) los cuales son solubles en medio ácido y los ácidos húmicos $(\mathrm{AH})$ que son insolubles en medio ácido. ${ }^{2-4}$

Estudios recientes indican que los ácidos húmicos están constituidos por clusters supramoleculares formados por el ensamblaje de bio-moléculas a través de enlaces químicos; los cuales se mantienen unidos por puentes de hidrogeno y por interacciones hidrofobicas y de van der Waals. ${ }^{5}$ Los ácidos húmicos están conformados por la unión de estructuras inalteradas de segmentos poliméricos de plantas y de fragmentos orgánicos con grupos del tipo carboxílico, fenólico, aldehído, cetonico, amido y amino. Esta composición estructural le confiere a los ácidos húmicos propiedades tales como su anfifilidad y su capacidad de intercambio catiónico. ${ }^{5,6}$

Se estima que aproximadamente un cuarto del peso molecular de las sustancias húmicas se debe a los grupos oxigenados, principalmente grupos carboxílicos que aumentan con el grado de humificación de la materia orgánica y que pueden formar carboxilatos con metales presentes en el medio, grupos fenólicos que se forman en los etapas iniciales de humificación y grupos carbonilos que por reacciones de oxidación originan grupos carboxílicos..$^{5,6}$

Las sustancias húmicas desempeñan un papel importante en la naturaleza, ya que debido a sus grupos funcionales oxigenados participan en procesos de retención de cationes que son esenciales para las plantas además de retener el calor en la superficie debido a su color oscuro. ${ }^{4}$ Entre los usos actuales de estas sustancias se destacan su actividad como fertilizantes, su capacidad para retener metales útiles para la agricultura y para dosificar el exceso de fertilizantes minerales y en la retención de sustancias toxicas para las plantas. ${ }^{1}$

Una fuente importante de sustancias húmicas son los carbones de bajo rango, los cuales poseen una estructura formada por anillos aromáticos, unidos por puentes del tipo alquileno, éster o éter y con cadenas laterales de grupos alquilo, carboxilo e hidroxilo, lo que les confiere una estructura bastante parecida a la de los ácidos húmicos. Esto posibilita la extracción con soluciones alcalinas del material húmico de este tipo de carbones. ${ }^{7,8}$

Los ácidos húmicos extraídos de carbón fósil difieren estructuralmente dependiendo del rango del carbón fósil y de la región de los yacimientos, sin embargo en términos generales los principales constituyentes de los ácidos húmicos son fragmentos aromáticos unidos a través de diversos tipos de puentes químicos y con sustituyentes ricos en oxígeno. ${ }^{9}$

Cuando los carbones fósiles se someten a reacciones de oxidación moderadas ocurre una degradación parcial de la materia orgánica presente en los carbones al tiempo en que se aumenta el número de grupos funcionales conteniendo oxígeno, principalmente los de tipo carboxílico y fenólico. Este tipo de reacciones incrementa el contenido de sustancias húmicas en su estructura, lo que también conlleva a mayores porcentajes de extracción de este tipo de materiales. ${ }^{7,8,10}$

En este estudio se tomó un carbón bajo rango proveniente de Montelíbano (Córdoba-Colombia) y se sometió a procesos de oxidación para aumentar el porcentaje de extracción de ácidos húmicos (AH).

\section{EXPERIMENTAL}

La muestra proveniente de la mina de Montelibano (CordobaColombia) fue triturada y tamizada a tamaño de partículas entre 3,1 y 7,1 mm. Posteriormente porciones de $100 \mathrm{~g}$ del material fueron desmineralizados durante $1 \mathrm{~h}$ a temperatura ambiente con ácido clorhídrico 0,5 M (Merck). Antes del proceso de oxidación el material de la muestra de carbón fue sometida a debitumizacion con una mezcla etanol-benceno (1:1 v/v) (Merck) en reflujo durante $24 \mathrm{~h}$. Posteriormente el solvente fue removido por destilación en Soxhlet y filtración al vacío con agua destilada. La humedad remanente se eliminó por secado a $80^{\circ} \mathrm{C}$ durante $12 \mathrm{~h}$. 


\section{Oxidación con aire}

Para la oxidación con aire se usaron $50 \mathrm{~g}$ de muestra y un flujo de aire de $100 \mathrm{~mL} / \mathrm{min}$ en un reactor de porcelana con un lecho fluidizado. Este proceso fue realizado en las temperaturas de 180, 200 y $220^{\circ} \mathrm{C}$.

\section{Oxidación acuosa}

La oxidación en medio acuoso fue realizada con peróxido de hidrogeno (Merck) y con ácido nítrico (Merck).

Para la oxidación con peróxido de hidrogeno se adicionaron en un erlenmeyer $5 \mathrm{~g}$ de carbón oxidado con aire, $30 \mathrm{~mL}$ de una solución al $30 \%$ (v/v) de $\mathrm{H}_{2} \mathrm{O}_{2}$ y 15 mL de ácido acético concentrado (Merck 99$100 \%$ ). Este sistema fue calentado a $60{ }^{\circ} \mathrm{C}$ y mantenido en agitación continua durante 6 y $12 \mathrm{~h}$.

La oxidación con ácido nítrico se realizó mezclando $5 \mathrm{~g}$ de carbón oxidado con aire con $30 \mathrm{~mL}$ de $\mathrm{HNO}_{3}$ al $30 \%$ (m/v). Este sistema fue calentado a $60{ }^{\circ} \mathrm{C}$ y mantenido en agitación continua durante 6 y $12 \mathrm{~h}$.

Para la extracción de los ácidos húmicos de los carbones oxidados, se agregaron a $5 \mathrm{~g}$ de estos materiales $100 \mathrm{~mL}$ de $\mathrm{NaOH}(0,1 \mathrm{M})$ (Sigma-Aldrich), manteniéndose este sistema durante 1 h en agitación continua. Posteriormente la solución se filtró al vacío y al filtrado se le adicionaron $100 \mathrm{~mL}$ de $\mathrm{HCl}(0,1 \mathrm{M})$ (Merck) para precipitar los ácidos húmicos. Este sistema se mantuvo en reposo durante 24 $\mathrm{h}$ y posteriormente fue centrifugado a $3600 \mathrm{rpm}$ durante $10 \mathrm{~min}$. El coloide obtenido después de la centrifugación fue lavado con porciones de etanol y finalmente se secó a $100{ }^{\circ} \mathrm{C}$ durante $1 \mathrm{~h}$ para ser sometido a diferentes análisis. El proceso de oxidación en solución acuosa fue realizado por triplicado en el caso de los dos agentes oxidantes, no siendo observadas desviaciones estándar mayores de $1,3 \%$ en ninguno de los casos.

\section{Caracterización de los materiales}

Los materiales fueron caracterizados a través de espectrometría en el infrarrojo (FTIR), análisis próximo, análisis elemental y cuantificación de grupos carboxílicos. El porcentaje de ácidos húmicos extraídos se cuantifico en relación a la masa de ácidos humicos extraída a partir del peso inicial de las muestras de carbón oxidadas (Ecuación 1)

$$
\left[\text { Masa }_{A H} / \text { Masa }_{\text {Carbon Oxidado }}\right] \times 100
$$

Los espectros FTIR fueron colectados en un equipo Perkin Elmer modelo 1600. Para este análisis las muestras se dispersaron en $\mathrm{KBr}$ en una relación muestra: $\mathrm{KBr}$ de 1:100, seguidamente por aplicación de presión se formaron pastillas circulares que fueron analizadas en el rango de 400 a $4500 \mathrm{~cm}^{-1}$, con una resolución de $2 \mathrm{~cm}^{-1}$.

Los análisis próximo y último fueron realizados por la empresa SGS de Barranquilla-Colombia.

Los ácidos carboxílicos fueron cuantificados a través del método de titulación sódica, descrito por Kononova. ${ }^{11}$

\section{RESULTADOS Y DISCUSIÓN}

En los espectros FTIR del carbón original (analizado en estudios previos realizados en el grupo de carboquimica) y del carbón oxidado con aire a diferentes temperaturas y tiempos (Figura 1), se observó que el carbón sin oxidar no presentó señales de grupos carboxílicos $\left(\sim 3400 \mathrm{~cm}^{-1}\right)$, sin embargo este material presentó una banda en 1640 $\mathrm{cm}^{-1}$ ocasionada por la vibración de estiramiento de enlaces del tipo $\mathrm{C}=\mathrm{C}$ aromático y probablemente estiramientos $\mathrm{C}=\mathrm{O}$. A pesar de que los carbones fósiles poseen en su estructura anillos aromáticos la banda a $3040 \mathrm{~cm}^{-1}$ no está presente en los espectros de infrarrojo, lo que indica que las estructuras aromáticas presentan un alto grado de condensación y de sustituciones. ${ }^{1,12}$

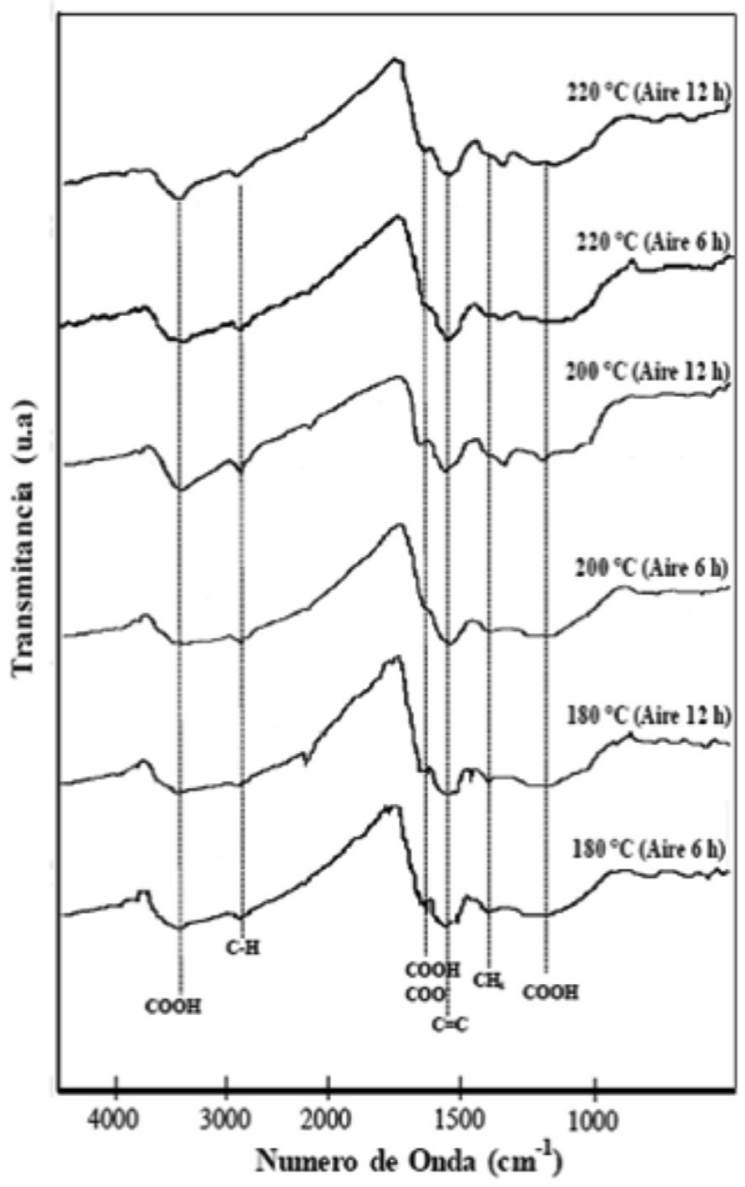

Figura 1. Espectros FTIR de carbones oxidados con aire a diferentes temperaturas y tiempos de reacción

En los carbones oxidados se observó una banda centrada en 3400 $\mathrm{cm}^{-1}$, la cual se debe al estiramiento $\mathrm{O}-\mathrm{H}$ de grupos carboxílicos o de alcoholes, lo cual confirma que la oxidación con aire condujo a la formación de estos grupos. Estos materiales también presentaron una banda a $2820 \mathrm{~cm}^{-1}$, atribuida a estiramientos $\mathrm{C}-\mathrm{H}$ de cadenas alifáticas o a grupos metileno, así como una banda a $1630 \mathrm{~cm}^{-1}$ ocasionada por estiramientos $\mathrm{C}=\mathrm{C}$ aromático. ${ }^{1,13}$ En estos materiales también se observó una banda en $1360 \mathrm{~cm}^{-1}$ que puede deberse a una flexión simétrica de un grupo metil ${ }^{13} \mathrm{y}$ una banda en $1580 \mathrm{~cm}^{-1}$ que puede ser asignada a grupos carboxilo $(\mathrm{COOH})$, carboxilato (-COO-) o del tipo éster (COOR). ${ }^{2,13,14}$ De igual manera la banda a $3040 \mathrm{~cm}^{-1}$ atribuida parte de la estructura aromática con poco condensación no fue observada en estos materiales, lo que indica que el proceso de oxidación no afecto esta parte de la estructura del carbón original.

Los resultados del análisis próximo (Tabla 1) muestran que la materia volátil aumenta al oxidarse el carbón original, observándose que el material obtenido al oxidar a $200{ }^{\circ} \mathrm{C}$ durante $12 \mathrm{~h}$ fue el que presentó mayor valor de este parámetro, el cual se asocia a la presencia grupos funcionales que se descomponen a bajas temperaturas, como es el caso de los grupos oxigenados, este hecho también se refleja en el menor porcentaje de carbono fijo presente en este material lo que indica que parte de la estructura rica en carbono del carbón original fue oxidada. El aumento del carbono fijo en todos los sólidos (excepto 
Tabla 1. Análisis próximo y porcentaje de extracción de ácidos húmicos del carbón original y oxidado con aire a diferentes temperaturas y tiempos

\begin{tabular}{|c|c|c|c|c|c|c|c|}
\hline Análisis próximo (\%) & Carbón original & $\begin{array}{c}180^{\circ} \mathrm{C} \\
6 \mathrm{~h}\end{array}$ & $\begin{array}{c}180^{\circ} \mathrm{C} \\
12 \mathrm{~h}\end{array}$ & $\begin{array}{c}200^{\circ} \mathrm{C} \\
6 \mathrm{~h}\end{array}$ & $\begin{array}{c}200{ }^{\circ} \mathrm{C} \\
12 \mathrm{~h}\end{array}$ & $\begin{array}{c}220^{\circ} \mathrm{C} \\
6 \mathrm{~h}\end{array}$ & $\begin{array}{c}220{ }^{\circ} \mathrm{C} \\
12 \mathrm{~h}\end{array}$ \\
\hline Humedad & 13,12 & 4,02 & 3,89 & 3,96 & 10,16 & 2,92 & 7,72 \\
\hline Ceniza & 2,78 & 3,77 & 3,70 & 3,70 & 3,43 & 3,46 & 3,49 \\
\hline Materia Volátil & 38,20 & 42,30 & 40,28 & 43,80 & 45,23 & 42,80 & 42,00 \\
\hline Carbono fijo & 45,90 & 49,91 & 52,13 & 48,54 & 41,18 & 50,82 & 46,79 \\
\hline
\end{tabular}

Tabla 2. Análisis elemental del carbón original y oxidado con aire a diferentes temperaturas y tiempos

\begin{tabular}{|c|c|c|c|c|c|c|c|}
\hline Análisis ultimo (\%) & $\begin{array}{c}\text { Carbón } \\
\text { Original }\end{array}$ & $\begin{array}{c}180^{\circ} \mathrm{C} \\
6 \mathrm{~h}\end{array}$ & $\begin{array}{c}180^{\circ} \mathrm{C} \\
12 \mathrm{~h}\end{array}$ & $\begin{array}{c}200^{\circ} \mathrm{C} \\
6 \mathrm{~h}\end{array}$ & $\begin{array}{c}200{ }^{\circ} \mathrm{C} \\
12 \mathrm{~h}\end{array}$ & $\begin{array}{c}220^{\circ} \mathrm{C} \\
6 \mathrm{~h}\end{array}$ & $\begin{array}{c}220^{\circ} \mathrm{C} \\
12 \mathrm{~h}\end{array}$ \\
\hline Carbono (C) & 67,65 & 68,49 & 67,67 & 68,34 & 68,04 & 68,44 & 68,10 \\
\hline Hidrogeno $(\mathrm{H})$ & 4,60 & 2,37 & 2,27 & 2,23 & 2,18 & 2,10 & 4,02 \\
\hline Nitrógeno (N) & 1,57 & 1,80 & 2,91 & 2,10 & 1,20 & 2,09 & 1,76 \\
\hline Azufre (S) & 0,60 & 0,54 & 0,52 & 0,52 & 0,53 & 0,49 & 0,60 \\
\hline Oxígeno $(\mathrm{O})$ & 25,58 & 26,8 & 26,63 & 26,81 & 28,05 & 26,88 & 25,52 \\
\hline Relación C/O & 2,64 & 2,56 & 2,54 & 2,55 & 2,42 & 2,55 & 2,69 \\
\hline Ácidos húmicos (\%) & 24,25 & 28,20 & 29,80 & 29,50 & 27,80 & 27,20 & 21,02 \\
\hline
\end{tabular}

el oxidado a $200{ }^{\circ} \mathrm{C}$ durante $12 \mathrm{~h}$ ) en relación al carbón original se debe principalmente a la disminución del porcentaje de humedad, el cual tiene un valor superior en este material y que puede estar asociado posiblemente a factores relacionados con el área superficial específica, en cuanto que en los sólidos oxidados parte de la humedad puede estar asociada al aumento del carácter hidrofílico inducido por la formación de grupos polares oxigenados.

A través del análisis último (Tabla 2) de los materiales obtenidos al oxidar el carbón original se evidencio el aumento de oxígeno en casi todos los sólidos, excepto en el material obtenido a $220{ }^{\circ} \mathrm{C}$ y $12 \mathrm{~h}$ en el que ocurrió la disminución de este elemento, lo que sugiere que la exposición del carbón a la condición más extrema de oxidación por más tiempo conduce a la descomposición de parte de los grupos oxigenados y de la estructura carbonosa a $\mathrm{CO}_{2}$ y $\mathrm{H}_{2} \mathrm{O}$. El contenido de carbono en estos materiales varía poco con las condiciones de preparación, en cuanto que los porcentajes de nitrógeno y azufre varían en función de la descomposición de los grupos oxigenados y de la posible formación de SOx y NOx durante la oxidación del carbón. La relación carbono/oxigeno disminuyo por el proceso de oxidación del carbón original a excepción del material oxidado por $12 \mathrm{~h}$ a $220{ }^{\circ} \mathrm{C}$, lo que confirma la perdida de grupos oxigenados en este material.

El porcentaje de extracción de AH a partir de las muestras de carbón oxidadas con aire fue mayor en la mayoría de los casos al porcentaje obtenido del carbón original (Tabla 1). Las mayores cantidades de AH fueron extraídas de los materiales oxidados a 180 y $200^{\circ} \mathrm{C}$, de los cuales se obtuvo un porcentaje superior al obtenido del carbón original. En el caso del solido tratado a $220^{\circ} \mathrm{C}$ durante $12 \mathrm{~h}$ el porcentaje de ácidos húmicos fue inferior al contenido en el carbón original, lo que indica que esas condiciones de tratamiento conducen a la descomposición de grupos funcionales oxigenados que contribuyen con la presencia de estructuras similares a los ácidos húmicos, lo que se confirmó por el menor contenido de oxígeno en este material.

En la Figura 2 se observa que la oxidación con $\mathrm{H}_{2} \mathrm{O}_{2}$ durante $6 \mathrm{~h}$ condujo al aumento de la cantidad de ácidos húmicos en la estructura de los carbones previamente oxidados con aire, notándose que el porcentaje de extracción fue superior al $30 \%$ en todos los casos. En estos solidos los mayores porcentajes de extracción se obtuvieron al pre-oxidar el carbón con aire durante 12 h, observándose también que en esos solidos el aumento de la temperatura condujo a la disminución del contenido de AH. En relación pre-oxidados durante $6 \mathrm{~h}$ con aire, el mayor contenido de $\mathrm{AH}$ se obtuvo del solido tratado a $200{ }^{\circ} \mathrm{C}$.

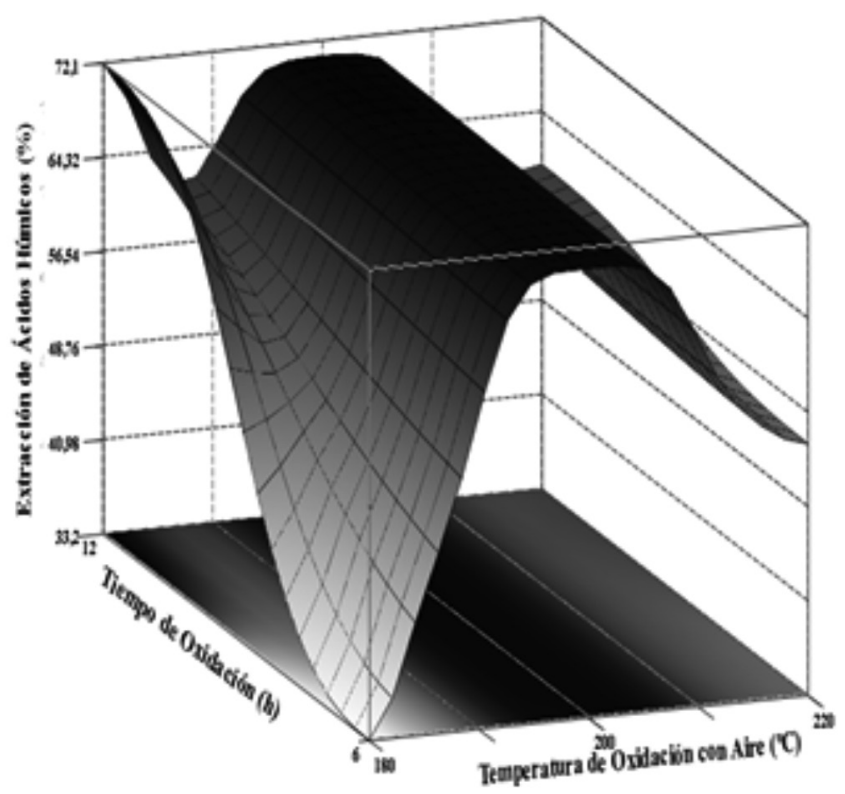

Figura 2. Porcentaje de extracción de ácidos húmicos de carbones oxidados con aire y con peróxido de hidrogeno durante 6 h en función de la temperatura y del tiempo de oxidación con aire

El contenido de $\mathrm{AH}$ en los materiales oxidados con $\mathrm{H}_{2} \mathrm{O}_{2}$ durante 12 h (Figura 3) decreció al aumentar la temperatura y al disminuir el tiempo de pre-oxidación con aire. En estos solidos de igual manera se observó que el porcentaje de extracción fue superior al 50\% en todos los casos. La mayor cantidad de ácidos húmicos extraídos de las muestras oxidadas con $\mathrm{H}_{2} \mathrm{O}_{2}$ se obtuvo del material tratado con este agente oxidante durante $12 \mathrm{~h}$ y pre-tratado con aire a $180{ }^{\circ} \mathrm{C}$ durante $12 \mathrm{~h}$, consiguiendo porcentajes del 75,3\% de extracción de ácidos húmicos. 


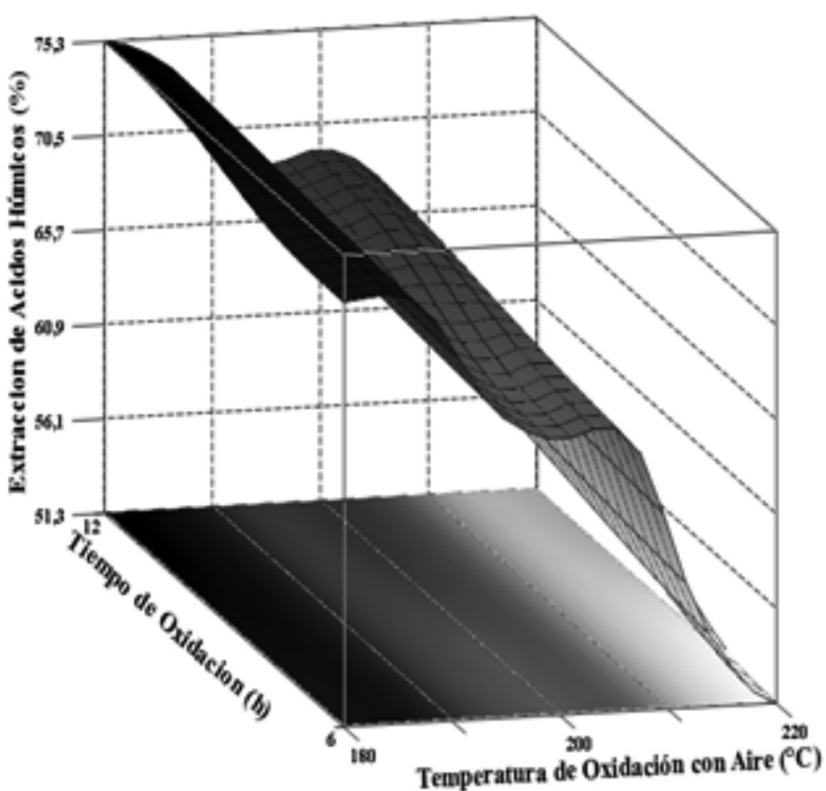

Figura 3. Porcentaje de extracción de ácidos húmicos de carbones oxidados con aire y con peróxido de hidrogeno durante 12 h en función de la temperatura y del tiempo de oxidación con aire

En las Figuras 4 y 5 se observa que los mayores porcentajes de ácidos húmicos extraídos de las muestras oxidadas con ácido nítrico durante 6 y $12 \mathrm{~h}$, se obtienen de los materiales pre-tratados con aire a $200{ }^{\circ} \mathrm{C}$ durante $12 \mathrm{~h}$. La forma de las superficies de respuesta en los dos casos presentan perfiles similares, sin embargo los sólidos oxidados con el ácido nítrico durante $12 \mathrm{~h}$ contienen mayor contenido de ácidos húmicos, consiguiéndose porcentajes de extracción de 85,3\% en el material pre-oxidado con aire a $200{ }^{\circ} \mathrm{C}$ por $12 \mathrm{~h}$.

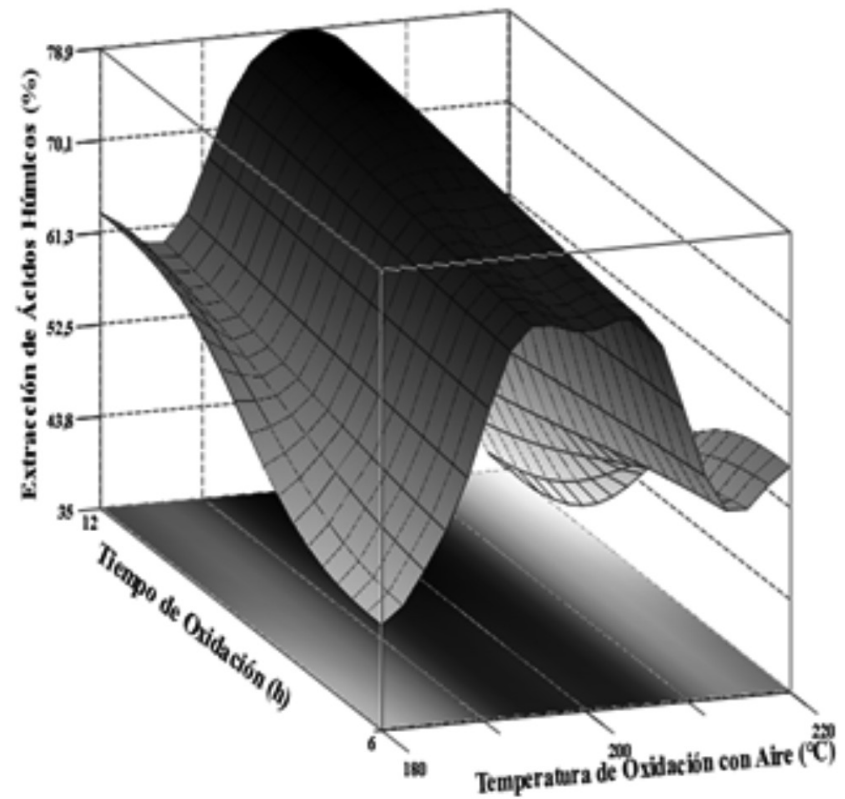

Figura 4. Porcentaje de extracción de ácidos húmicos de carbones oxidados con aire y con ácido nítrico durante 6 h en función de la temperatura y del tiempo de oxidación con aire

Los espectros de los ácidos húmicos obtenidos después de la oxidación con peróxido de hidrogeno (Figura 6), presentaron una banda ancha centrada en $3400 \mathrm{~cm}^{-1}$ la cual es característica de grupos $\mathrm{OH}$ presentes en grupos carboxílicos. ${ }^{1}$ Esta banda en este tipo de

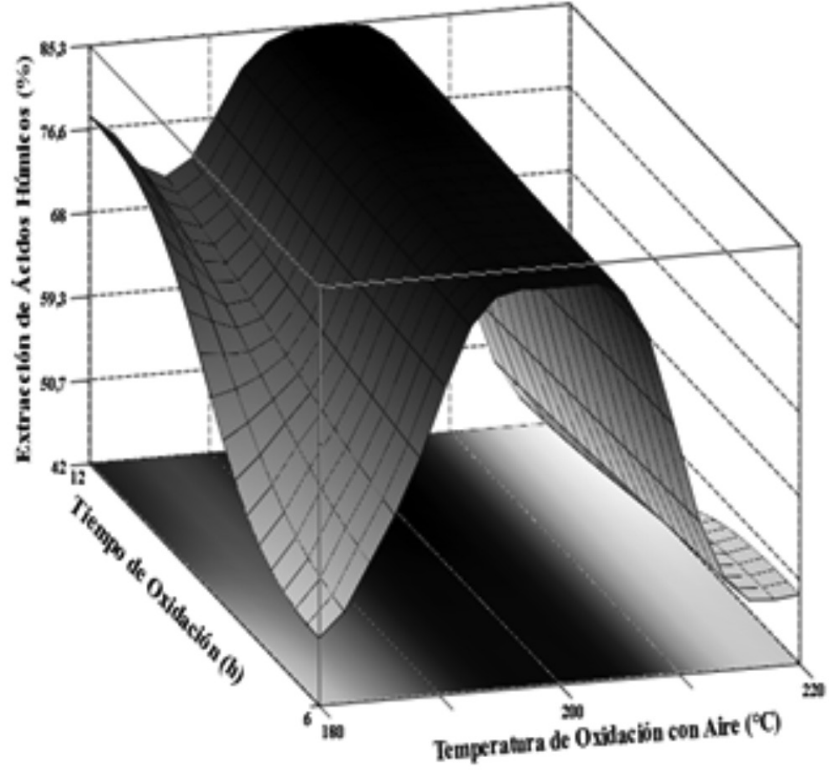

Figura 5. Porcentaje de extracción de ácidos húmicos de carbones oxidados con aire y con ácido nítrico durante 12 h en función de la temperatura y del tiempo de oxidación con aire

materiales es más ancha que la registrada por lo materiales apenas oxidados con aire, lo que indica el aumento de grupos carboxílicos. También se observó el incremento de bandas estrechas entre 3000 y $2800 \mathrm{~cm}^{-1}$, las cuales son atribuidas a enlaces C-H en estructuras alifáticas, lo que sugiere que durante el proceso de oxidación con peróxido hubo rompimiento de macroestructuras para originar la presencia de grupos alifáticos que pueden estar como sustituyentes en estructuras aromáticas o como puentes entre ellas. Además de estas bandas se observaron otras a $1720 \mathrm{~cm}^{-1}(\mathrm{C}=\mathrm{O}), 1270 \mathrm{~cm}^{-1}(\mathrm{C}=\mathrm{C}$ o Ar-O-R) y $1300-1000 \mathrm{~cm}^{-1}$ (C-O-C).$^{1,12-15}$

En el caso de los ácidos húmicos obtenidos al oxidar con ácido nítrico (Figura 7) se observaron las mismas bandas registradas en los materiales obtenidos al oxidar con el peróxido de hidrogeno, con la diferencia de la presencia de bandas a 1540 y $1370 \mathrm{~cm}^{-1}$ originadas por la presencia del estiramiento simétrico $\mathrm{N}=\mathrm{O}$ de los grupos nitro formados principalmente en estructuras aromáticas por la oxidación con el ácido nítrico. ${ }^{16,17}$

Los valores del número de grupos carboxílicos (Tabla 3) en los ácidos húmicos obtenidos al oxidar con agentes químicos indican que en el caso de los tratados con peróxido de hidrogeno, las condiciones más suaves de oxidación con aire $\left(180-200{ }^{\circ} \mathrm{C}\right)$ condujo a solidos con mayor número de ácidos carboxílicos. El tratamiento a temperaturas superiores especialmente con el mayor tiempo de tratamiento con la solución de peróxido de hidrogeno conducen a la formación de $\mathrm{AH}$ con menor número de grupos carboxílicos o a $\mathrm{AH}$ en los cuales el mayor tiempo de exposición al agente oxidante condujo a la descomposición de grupos oxigenados, entre ellos los carboxílicos a $\mathrm{CO}_{2}$. Entre estos solidos fue el material obtenido del carbón oxidado con aire a $180{ }^{\circ} \mathrm{C}$ por $12 \mathrm{~h}$ y con peróxido de hidrogeno por $12 \mathrm{~h}$ el que mostro mayor número de grupos carboxílicos.

En relación a los AH obtenidos después de la reacción de oxidación con ácido nítrico se observó que estos solidos presentan, en general mayor número de ácidos carboxílicos que los obtenidos con peróxido de hidrogeno en iguales condiciones de pre-tratamiento, lo cual explica los mayores porcentajes de extracción de $\mathrm{AH}$ obtenidos en estos materiales, sin embargo el ácido nítrico además de conducir a reacciones de oxidación de parte de la estructura carbonosa, contribuyó también con la formación de grupos nitro en la estructura. 


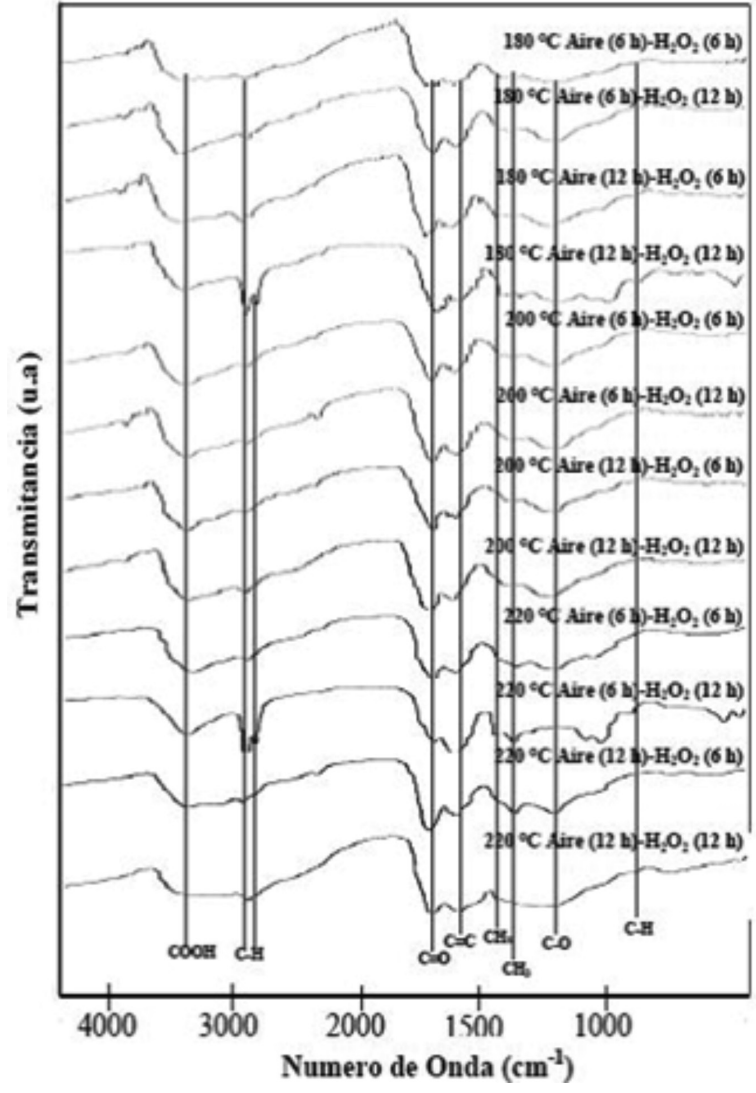

Figura 6. Espectros FTIR de ácidos húmicos extraídos de carbón oxidado con aire $\left(180,200\right.$ y $220^{\circ} \mathrm{C}$ por 6 y $\left.12 \mathrm{~h}\right)$ y con peróxido de hidrogeno $(6$ y $12 \mathrm{~h})$

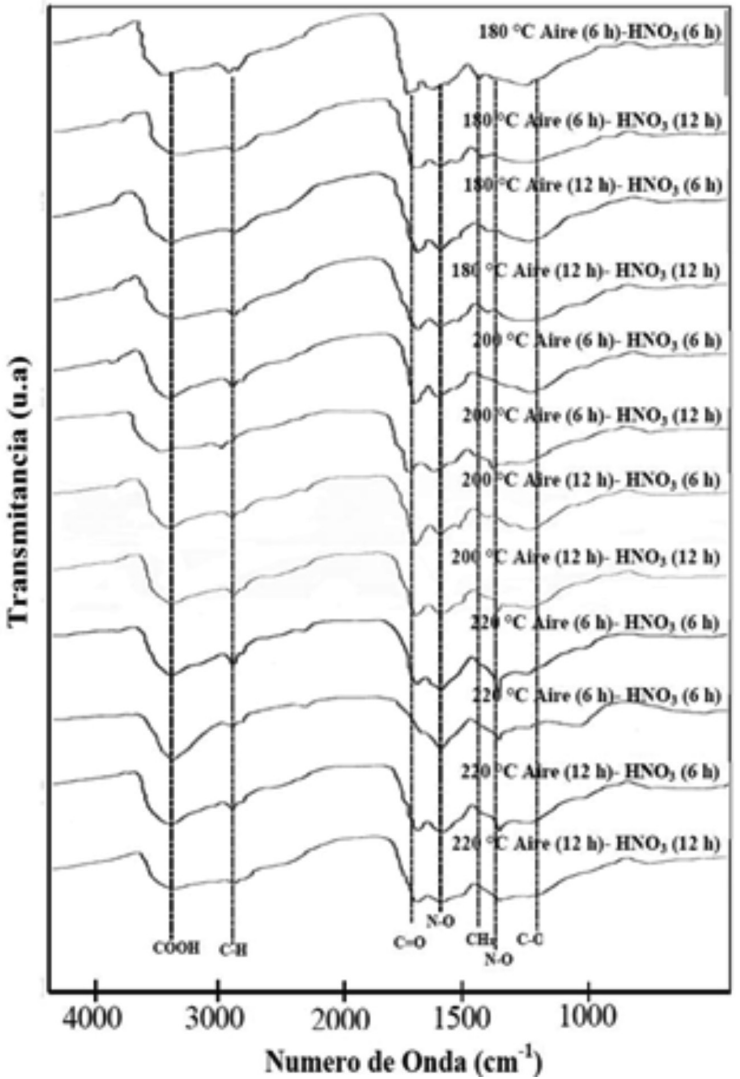

Figura 7. Espectros FTIR de ácidos húmicos extraídos de carbón oxidado con aire $\left(180,200\right.$ y $220^{\circ} \mathrm{C}$ por 6 y $12 \mathrm{~h}$ ) y con ácido nítrico $(6$ y $12 \mathrm{~h})$

Tabla 3. Número de grupos carboxílicos de los ácidos húmicos obtenidos al oxidar la muestra de carbón original con aire, $\mathrm{H}_{2} \mathrm{O}_{2}$ y $\mathrm{HNO}_{3}$

\begin{tabular}{ccccccc}
\hline $\begin{array}{c}\text { Número de grupos } \\
\text { carboxílicos (meq/) }\end{array}$ & $\begin{array}{c}\text { Oxidación } \\
180-6 \mathrm{~h}\end{array}$ & $\begin{array}{c}\text { Oxidación } \\
180-12 \mathrm{~h}\end{array}$ & $\begin{array}{c}\text { Oxidación } \\
200-6 \mathrm{~h}\end{array}$ & $\begin{array}{c}\text { Oxidación } \\
200-12 \mathrm{~h}\end{array}$ & $\begin{array}{c}\text { Oxidación } \\
220-6 \mathrm{~h}\end{array}$ & $\begin{array}{c}\text { Oxidación } \\
220-12 \mathrm{~h}\end{array}$ \\
\hline $\mathrm{H}_{2} \mathrm{O}_{2}-6 \mathrm{~h}$ & 3,48 & 4,17 & 4,03 & 3,22 & 2,77 & 2,65 \\
$\mathrm{H}_{2} \mathrm{O}_{2}-12 \mathrm{~h}$ & 3,57 & 4,28 & 3,97 & 2,95 & 2,70 & 3,62 \\
$\mathrm{HNO}_{3}-6 \mathrm{~h}$ & 3,97 & 4,26 & 4,38 & 4,57 & 4,58 & 3,32 \\
$\mathrm{HNO}_{3}-12 \mathrm{~h}$ & 4,10 & 4,27 & 4,42 & 4,82 & 3,22 \\
\hline
\end{tabular}

\section{CONCLUSIONES}

La oxidación con aire aumentó el contenido de oxígeno en la estructura del carbón original, lo cual condujo al aumento del porcentaje de extracción de ácidos húmicos después de este proceso inicial de oxidación.

La mayor cantidad de ácidos húmicos extraídos de las muestras oxidadas con $\mathrm{H}_{2} \mathrm{O}_{2}$ se obtuvo del material tratado con este agente oxidante durante $12 \mathrm{~h}$ y pre-tratado con aire a $180{ }^{\circ} \mathrm{C}$ durante $12 \mathrm{~h}$, consiguiendo extraerse el 75,3\% de ácidos húmicos. En el caso de los materiales oxidados con ácido nítrico el mayor extracción se obtuvo del material oxidado a $200{ }^{\circ} \mathrm{C}$ por $12 \mathrm{~h}$ con aire y con ácido nítrico durante $12 \mathrm{~h}(85,3 \%)$.

Los espectros FTIR de los ácidos húmicos obtenidos al tratar con las soluciones acuosas oxidantes mostraron la presencia de bandas relacionadas con grupos oxigenados y con estructuras alifáticas, lo que indica que además de reacciones de oxidación también ocurrieron otras en las que probablemente ocurrió el rompimiento de estructuras cíclicas y aromáticas que contribuyeron con el aparecimiento de estas bandas.

El aumento de ácidos húmicos después de la re-oxidación con peróxido de hidrogeno y ácido nítrico se relacionó directamente con el aumento de grupos carboxílicos, lo que indica que estos agentes contribuyen con la formación de estos grupos, sin embargo en las condiciones más extremas de tratamiento parte de estos grupos puede descomponerse a especies como $\mathrm{CO}_{2}$.

\section{REFERENCIAS}

1. Proidakov, A. G.; Solid Fuel Chem. 2009, 43, 9.

2. Peuravuori, J.; Žbánková, P.; Pihlaja, K.; Fuel Process Technol. 2006, 87,829 .

3. Butuzova, L.; Krzton, A.; Bazarovaar, O.; Fuel 1998, 77, 581.

4. Navarro, S.; Navarro, G.; Química agrícola: el suelo y los elementos químicos esenciales para la vida, $1^{\text {th }}$ ed., Mundi-prensa: Madrid, 2003.

5. Salati, S.; Papa, G.; Adani, F.; BioTechnol. Adv. 2011, 29, 913.

6. Nebbioso, A.; Piccolo, A.; Anal. Chim. Acta 2012, 720, 77

7. Rhoads, C.; Senftle, J.; Coleman, M.; Davis, A.; Painter, P.; Fuel 1983, $62,1387$.

8. Calemma, V.; Iwanski, P.; Rausa, R.; Girardi, E.; Fuel 1994, 73, 700.

9. Kwiatkowska, J.; Provenzano, M.; Senesi, N.; Geoderma 2008, 148, 200 . 
10. Patrakov, Y. F.; Schastlivtsev, E. L.; Mandrov, G. A.; Solid Fuel Chem. 2010, 44, 293

11. Kononova, M.; Soil Organic Matter, $1^{\text {st }}$ ed., Pergamon Press: Oxford, 1966.

12. Ibarra, J.; Muñoz, E.; Moliner, R.; Org. Geochem. 1996, 24, 725.

13. Wang, S.; Tang, Y.; Schobert, H.; Guo, Y.; Su, Y.; Energy Fuels 2011, 25,5672 .
14. Sonibare, O.; Haeger, T.; Foley S.; Energy 2010, 35, 5347.

15. Verheyen, V.; Rathbone, R.; Jagtoyen, M.; Derbyshire, F.; Carbon 1995 , $33,763$.

16. Alvarez, R.; Clemente, C.; Gomez-Limon, D.; Fuel 2003, 82, 2007.

17. Nasir, S.; Sarfaraz, T.; Verheyen, T.; Chaffee, A.; Fuel. Process Technol. 2011, 92, 983. 